\title{
UTILIZAÇÃO DE TÉCNICAS DE RESPIRAÇÃo E RELAXAMENTO PARA ALÍVIO DE DOR E ANSIEDADE NO PROCESSO DE PARTURIÇÃO
}

\author{
Nilza Alves Marques Almeida ${ }^{1}$ \\ Joaquim Tomé de Sousa ${ }^{2}$ \\ Maria Márcia Bachion² \\ Nusa de Almeida Silveira ${ }^{3}$
}

Almeida NAM, Sousa JT, Bachion MM, Silveira NA. Utilização de técnicas de respiração e relaxamento para alívio de dor e ansiedade no processo de parturição. Rev Latino-am Enfermagem 2005 janeiro-fevereiro; 13(1):52-8.

Objetivou-se avaliar o efeito de técnicas de respiração e de relaxamento sobre a dor e a ansiedade, na parturição. Dezessete primigestas (grupo controle - GC) receberam assistência de rotina e dezenove (grupo experimental - GE) foram orientadas e estimuladas a realizar técnicas de respiração e relaxamento. Avaliou-se a dor, através da escala analógica visual, e a ansiedade, através dos inventários de ansiedade-traço e estado. A intensidade de dor aumentou com a evolução do trabalho de parto para ambos os grupos. O nível de ansiedade na fase latente foi baixo para ambos os grupos; na fase ativa foi médio para o GC e baixo para o GE; na fase de transição foi médio e, no pós-parto, imediato, foi baixo, para ambos os grupos. Concluiu-se que as técnicas utilizadas não reduziram a intensidade de dor, mas promoveram ao GE a manutenção de nível baixo de ansiedade por maior tempo da parturição.

DESCRITORES: enfermagem; respiração; relaxamento; dor; ansiedade; parto

\section{THE USE OF RESPIRATION AND RALAXATION TECHNIQUES FOR PAIN AND ANXIETY RELIEF IN THE PARTURITION PROCESS}

This study aimed to evaluate the effect of respiration and relaxation techniques on pain and anxiety during labor. Seventeen parturients (Control Group - CG) received routine care and nineteen (Experimental Group -EG) were orientated and stimulated to perform respiration and relaxation techniques. Pain was evaluated by means of the visual analogy scale and anxiety by means of the anxiety inventories - trait and state. Pain intensity increased along with the evolution of the delivery for both groups. In the latent phase, anxiety levels were low for both groups; in the active phase, levels were average for $C G$ and low for $E G$. In the transition phase, levels were average and, in the immediate post-labor phase, low for both groups. It was concluded that the techniques used did not reduce pain intensity, but provided EG with lower anxiety levels for a longer time during labor.

DESCRIPTORS: nursing; respiration; relaxation; pain; anxiety; parturition

\section{UTILIZACIÓN DE TÉCNICAS DE RESPIRACIÓN Y RELAJACIÓN PARA EL ALIVIO DEL DOLOR Y DE LA ANSIEDAD EN EL PROCESO DE PARTO}

La finalidad de este estudio fue evaluar el efecto de técnicas de respiración y relajación sobre el dolor y la ansiedad durante el parto. Diecisiete primerizas (grupo control-GC) recibieron asistencia de rutina y diecinueve (grupo de experimento-GE) fueron orientadas y estimuladas a realizar técnicas de respiración y relajación. Se evaluó el dolor a través de la escala analógica visual y la ansiedad a través de los inventarios de ansiedad-apariencia y estado. La intensidad del dolor aumentó con la evolución del trabajo de parto para ambos grupos. El nivel de la ansiedad, en la fase latente, fue bajo para ambos grupos; en la fase activa fue medio para el GC y bajo para el GE; en la fase de transición fue medio y, en el post parto inmediato, bajo para ambos grupos. Se llegó a la conclusión de que las técnicas utilizadas no redujeron la intensidad del dolor, pero sí promovieron al GE el mantenerse en un nivel bajo de ansiedad por mayor tiempo en el parto.

DESCRIPTORES: enfermería; respiración; relajación; dolor; ansiedad; parto

\footnotetext{
${ }^{1}$ Professor Assistente, e-mail: nilza@fen.ufg.br; ${ }^{2}$ Professor Titular, e-mail: tome@ ufgvirtual.ufg.br; mbachion@fen.ufg.br. Faculdade de Enfermagem da Universidade Federal de Goiás; ${ }^{3}$ Professor Adjunto do Instituto de Ciências Biológicas da Universidade Federal de Goiás, e-mail: nusa@icb2.ufg.br
} 


\section{INTRODUÇÃO}

A dor é conceituada pela International Association for the Study of Pain, como experiência sensorial e emocional desagradável, associada a um dano real ou potencial dos tecidos, ou descrita em termos de tais lesões ${ }^{(1-2)}$.

A modulação da dor é realizada por mecanismos analgésicos endógenos, caracterizados por uma via pela qual a informação dolorosa percorre o sistema nervoso até o cérebro. $O$ agente causador de dor é detectado pelos nociceptores, axônios de células nervosas situadas na medula espinhal, que levam a informação dolorosa de sua origem periférica ao sistema nervoso central. No cérebro essa informação é identificada e se transforma em sensação de dor ${ }^{(3)}$.

A teoria de dor mais abrangente e aceita no momento é a Gate Control, elaborada por Melzack e Wall, em 1965, que considera tanto os aspectos fisiológicos como psicológicos da dor e contribui para sua compreensão, avaliação e controle. Segundo essa teoria, os impulsos nervosos evocados por lesões são influenciados na medula por outras células nervosas que atuam como portões, impedindo ou facilitando a passagem dos impulsos ${ }^{(4)}$.

A dor, durante a parturição, é uma resposta fisiológica, complexa, subjetiva e multidimensional aos estímulos sensoriais gerados, principalmente, pela contração uterina ${ }^{(2)}$. As outras causas fisiológicas da dor são a hipóxia da musculatura uterina, o estiramento cervical, vaginal e perineal durante o período expulsivo ${ }^{(3,5)}$, o estresse (níveis aumentados de glicocorticóides e catecolaminas) e o limiar baixo de tolerância à dor (baixos níveis de endorfina, fadiga e doença) ${ }^{(5-6)}$.

Como a interpretação dolorosa envolve aspectos sensitivos, cognitivos, comportamentais e culturais, torna-se necessária avaliação sistematizada $^{(7-8)}$.

Existem várias escalas para avaliação de dor como a escala analógica visual (Visual Analogue Scale-VAS), as escalas numéricas, as escaldas de categorias de expressões verbais e de representação gráfica não numérica (de faces, copos, cores, dentre outros). Elas são unidimensionais e não avaliam outras variáveis (padrão cultural, experiências pregressas, significado das situações, personalidade, atenção, emoções, contingência de reforço) que modificam a expressão de dor ${ }^{(9)}$.

A VAS é uma escala de fácil compreensão ao paciente. É provida de instruções claras e conceitos simples, permitindo aplicação rápida com intervenção mínima ${ }^{(10)}$. Consiste em uma régua de dez centímetros de comprimento que, em uma extremidade, tem as palavras "sem dor" e, na outra, "a pior dor imaginável". Preso à régua há um indicador móvel. O cliente indica a intensidade de sua dor posicionando o indicador, exatamente no local em que considera estar a intensidade de sua dor. A pontuação da intensidade de dor referida pelo cliente é lida numa reta graduada presente no verso da régua e pode assumir valores de zero a dez ${ }^{(9)}$.
Alguns estudiosos apontam uma relação entre ansiedade, medo e dor na parturição. Para eles, quando a dor é abolida a ansiedade também é aliviada ${ }^{(11)}$.

A ansiedade pode ser conceituada de duas formas distintas: ansiedade-traço e ansiedade-estado. A ansiedade-traço refere-se às diferenças individuais relativamente estáveis, de propensão ou tendência do indivíduo para vivenciar a ansiedade. A ansiedade-estado é uma condição transitória de tensão diante de uma circunstância percebida como ameaçadora, sem identificar-se o objeto de perigo, ou seja, simbólica, inespecífica e antecipada ${ }^{(12)}$.

Para avaliação do Traço de Ansiedade (Trait Anxiety) e Estado de Ansiedade (State Anxiety) foi criado, originalmente, o StateTrait Anxiety Inventory (STAI) que, no Brasil, é denominado Inventário de Ansiedade Traço-Estado (IDATE) ${ }^{(12)}$.

O IDATE determina o grau de ansiedade. Ele contém vinte afirmações com quatro possibilidades de concordância. O indivíduo responde a cada afirmativa do inventário avaliando a si próprio numa escala de quatro pontos ${ }^{(12)}$. Tanto na escala ansiedade-traço como ansiedade-estado, a pontuação pode variar de vinte (todas as respostas com valor 1) a oitenta (todas as respostas com valor 4). A pontuação de 20 a 40 indica baixo grau de ansiedade, maior que 40 a 60 indica médio grau de ansiedade e maior que 60 a 80 indica alto grau de ansiedade ${ }^{(13)}$.

Além da avaliação do grau de ansiedade, é possível avaliar sua intensidade. Para isso, desenvolveu-se uma escala crescente que permite pontuação de 0 a 10, na qual o indivíduo avaliado indica a intensidade com que sente cada uma das vinte afirmativas do IDATE. A somatória dessa pontuação pode assumir valores de vinte a duzentos. A pontuação de 20 a 80 indica baixa intensidade de ansiedade, mais de 80 a 140 pontos indica média intensidade de ansiedade e pontuação maior que 140 a 200 indica alta intensidade de ansiedade ${ }^{(13)}$.

A interação das variáveis grau e intensidade de ansiedade numa nova variável (nível de ansiedade) pode compensar as variações em diferentes indivíduos, uma vez que há a possibilidade da ocorrência de indivíduos com elevado grau de ansiedade e baixa intensidade ou vice-versa. A variável nível de ansiedade pode assumir valores de vinte a oitocentos pontos. A pontuação de 20 a 280 indica nível baixo de ansiedade, mais de 280 , a 540 pontos indica nível médio de ansiedade e pontuação maior de 540, a 800 indica nível alto de ansiedade ${ }^{(13)}$.

Pesquisadores de outros países têm estudado esses parâmetros durante o parto, buscando compreender aspectos relativos à fisiologia. Ao avaliarem o estado de ansiedade e a dor subjetiva antes, durante e após o trabalho de parto, através da VAS e do IDATE, verificaram que ocorre aumento da ansiedade e da dor com a progressão do trabalho de parto ${ }^{(14)}$. Outros autores têm estudado a natureza da experiência da dor durante o processo de parturição ${ }^{(2,8,15)}$. Diante das evidências da literatura de que a parturição é 
uma experiência que acarreta dor e ansiedade, estudos em nosso contexto sociocultural devem ser implementados, buscando compreender os fatores que interferem nesses fenômenos, para que 0 enfermeiro e outros profissionais de saúde envolvidos na assistência à parturiente possam intervir sobre eles.

Com a finalidade de contribuir para a melhoria da qualidade da assistência de enfermagem à parturiente, assim como ser referência para a avaliação da dor e da ansiedade no processo de parturição, elegeu-se como objetivo geral desse estudo avaliar o efeito de técnicas de respiração e de relaxamento sobre a dor e a ansiedade de parturientes durante o processo de parturição. Assim, estabeleceu-se como objetivos específicos:

1. verificar a intensidade de dor referida pelas parturientes durante as três fases do trabalho de parto;

2. avaliar o grau, a intensidade e o nível de ansiedade das parturientes durante as três fases do trabalho de parto e no pós-parto imediato;

3. estudar a correlação entre ansiedade e dor nas diferentes fases do trabalho de parto;

4. comparar a duração do trabalho de parto e as intensidades da dor e da ansiedade entre um grupo de parturientes que recebeu assistência "de rotina" e outro, que recebeu orientações e estímulo para a execução de técnicas de respiração e relaxamento durante a parturição.

\section{MATERIAL E MÉTODO}

A pesquisa de campo experimental foi realizada na enfermaria de pré-parto, no centro obstétrico e na enfermaria de pós-parto de uma maternidade pública, localizada na cidade de Goiânia, Goiás.

O projeto foi aprovado pela Direção Clínica da Maternidade e pelo Comitê de Ética em Pesquisa Médica Humana e Animal do Hospital das Clínicas da Universidade Federal de Goiás.

A amostra constituiu-se de parturientes admitidas para assistência ao parto, pelo Sistema Único de Saúde, no período de maio de 2000 a março de 2001.

Os critérios de inclusão foram: ser primigesta, evoluir para trabalho de parto normal e de baixo risco, estar na fase latente do trabalho de parto (até quatro centímetros de dilatação cervical) na admissão, não ser portadora de nenhuma patologia associada a complicações obstétricas e não ter participado de grupos de preparo psicoprofilático para o parto.

Constituíram-se em fatores para exclusão: a ocorrência de distócias da contratilidade uterina que necessitassem de oxitocina exógena, a ocorrência de distócias de trajeto, sofrimento fetal agudo, patologias ou intercorrências obstétricas com indicação de cesariana, a necessidade de realização de parto fórceps ou do uso de analgesia.

As parturientes foram alocadas por amostragem aleatória simples através de sorteio, com auxílio de uma moeda, em grupo controle (cara) e grupo experimental (coroa). Essa forma de sorteio foi eleita por ser de fácil aplicabilidade e entendimento para a parturiente. À medida que ocorria uma exclusão para o grupo sorteado, a próxima parturiente era incluída no mesmo grupo.

As parturientes do grupo controle $(\mathrm{GC})$ receberam a assistência de enfermagem de rotina da Maternidade e as do grupo experimental (GE) receberam assistência de enfermagem individualizada, com orientação e estímulo à utilização de técnicas de respiração e relaxamento durante o processo de parturição.

Utilizou-se, para coleta de dados, três protocolos: registro de dados da admissão, escalas de auto-avaliação de traço e estado de ansiedade $^{(12-13)}$ e escala analógica visual (VAS) ${ }^{(9)}$, para a avaliação da intensidade da dor. A VAS foi aplicada no início das fases latente, ativa e de transição, no momento em que a parturiente estava apresentando dor proveniente da contração uterina. A escala do traço de ansiedade foi aplicada na fase latente do trabalho de parto e a de estado de ansiedade nas fases ativa e de transição e no pós-parto imediato, a fim de comparar o nível de ansiedade apresentado pelas parturientes na admissão, durante a evolução do trabalho de parto e pós-parto imediato.

As técnicas de respiração e relaxamento utilizadas foram adaptadas dos métodos psicoprofiláticos de Dick Read ${ }^{(16)}$ e Fernand Lamaze $^{(6)}$ e aplicadas a partir da admissão da parturiente até 0 ato do parto da seguinte forma:

1. técnica de respiração: foi utilizada durante as contrações nas diferentes fases do trabalho de parto e no período expulsivo.

- fase latente: respiração total (respiração tóraco-abdominal lenta, com inspiração e expiração profundas, num ritmo natural);

- fase ativa: respiração torácica lenta (respiração lenta, com inspiração e expiração profundas e longas, num ritmo natural, direcionando a respiração para a região torácica);

- fase de transição: respiração de pressão sem execução de força de pressão abdominal (respiração lenta, com inspiração profunda sustentada por maior tempo durante o puxo contrátil, a fim de manter 0 diafragma exercendo força sobre o útero, seguido de expiração longa); - período expulsivo: respiração de pressão com execução de força abdominal (contração da musculatura estriada) no momento dos puxos; 2. técnica de relaxamento: soltura de toda a musculatura corporal associada à respiração total, nos intervalos das contrações uterinas.

Pela natureza das técnicas propostas, não é necessário preparo físico e condicionamento respiratório prévio da parturiente, por respeitar-se nas duas primeiras fases do trabalho de parto o ritmo respiratório natural e, na terceira, estimular-se uma pausa entre a inspiração profunda sustentada e a expiração longa, não oferecendo risco de hiperventilação.

Após a admissão, a parturiente era convidada a participar do estudo. Em caso de concordância, era feita a leitura explicativa do 
Termo de Consentimento Livre e Esclarecido e, logo após, procediase à assinatura do mesmo. Esse procedimento ocorria na presença de uma testemunha (um familiar ou a Chefe ou a Supervisora de enfermagem da maternidade). Em seguida realizava-se o sorteio para os grupos.

A partir desse momento, eram realizados os procedimentos de coleta de dados e o acompanhamento da evolução do trabalho, para ambos os grupos, e a assistência direta com orientação e incentivo do uso das técnicas de respiração e relaxamento ao grupo experimental.

Os dados foram analisados através de procedimentos de estatística descritiva, sendo apresentados em forma de média $(\chi) \pm$ desvio padrão ( $d p)$ e em percentual e foram submetidos aos testes $t$ de Student, Mann-Whitney e de correlação de Pearson, adotando-se o valor de $p<0,05$.

\section{RESULTADOS E DISCUSSÃO}

Todas as parturientes que atenderam aos critérios de inclusão aceitaram participar do estudo. Obteve-se inicialmente a participação de sessenta e cinco parturientes, sendo que vinte e nove $(44,62 \%)$ foram excluídas (doze por uso de ocitocina exógena, duas por parto fórceps e quinze por parto cesariano). Dessa forma, a amostra do estudo constou de trinta e seis parturientes, sendo dezessete do grupo controle (GC) e dezenove do grupo experimental (GE), apresentando média de idade igual a 20,06 e 21,30 anos, respectivamente.

Duração do trabalho de parto

Em relação à evolução do trabalho de parto, $0 \mathrm{GC}$ apresentou $339,70( \pm 168,45)$ minutos e o GE 445,26 $( \pm 158,05)$ minutos de duração média total de trabalho de parto; o GC apresentou 84,70 ( \pm $37,10)$ minutos e o GE 145,26 ( \pm 96,57) de duração média da fase latente; o GC apresentou 151,76 ( $\pm 129,23)$ e o GE 173,68 $( \pm 77,18)$ de duração média da fase ativa; e o GC apresentou 103,23 $( \pm 43,04)$ e 0 GE 126,31( $\pm 54,28)$ de duração média da fase de transição. Utilizando-se 0 teste $t$ de Student $(p<0,05)$ para comparar os dois grupos, não se encontrou diferença significativa quanto à duração total do trabalho de parto $(p=0,06)$, duração da fase ativa $(p=0,54)$ e de transição $(p=0,16)$ do trabalho de parto. Encontrou-se diferença estatisticamente significativa na fase latente $(p=0,01)$, sendo a duração maior para o GE.

Dor

Os resultados da avaliação da intensidade de dor sentida pelas parturientes durante as três fases do trabalho de parto, obtidos através da aplicação de escala analógica visual, estão apresentados na Tabela 1.

Tabela 1 - Intensidade da dor pontuada pelas parturientes do grupo controle (GC) e grupo experimental (GE) na escala analógica visual, durante as fases do trabalho de parto. Goiânia, GO. 2000/2001

\begin{tabular}{|c|c|c|c|c|c|c|}
\hline \multirow{3}{*}{$\begin{array}{c}\text { Pontuação } \\
\text { da } \\
\text { intensidade } \\
\text { de dor } \\
\end{array}$} & \multicolumn{6}{|c|}{ Fases do trabalho de parto } \\
\hline & \multicolumn{2}{|c|}{ Latente } & \multicolumn{2}{|c|}{ Ativa } & \multicolumn{2}{|c|}{ Transição } \\
\hline & GC (\%) & GE (\%) & $\mathrm{GC}(\%)$ & GE (\%) & $\mathrm{GC}(\%)$ & GE (\%) \\
\hline 1 & 0 & 0 & 0 & 0 & 0 & 0 \\
\hline 2 & 35,29 & 21,05 & 0 & 0 & 0 & 0 \\
\hline 3 & 17,64 & 15,79 & 0 & 0 & 0 & 0 \\
\hline 4 & 29,40 & 31,58 & 5,89 & 0 & 0 & 0 \\
\hline 5 & 5,89 & 21,05 & 5,89 & 5,26 & 0 & 0 \\
\hline 6 & 5,89 & 10,53 & 29,40 & 15,79 & 0 & 0 \\
\hline 7 & 5,89 & 0 & 23,53 & 31,58 & 5,89 & 5,26 \\
\hline 8 & 0 & 0 & 23,53 & 31,58 & 5,89 & 5,26 \\
\hline 9 & 0 & 0 & 11,76 & 10,53 & 17,64 & 21,05 \\
\hline 10 & 0 & 0 & 0 & 5,26 & 70,58 & 68,42 \\
\hline Total & 100,0 & 100,0 & 100,0 & 100,0 & 100,0 & 100,0 \\
\hline
\end{tabular}

Na fase latente do trabalho de parto houve predominância das pontuações de intensidade de dor de $2 \mathrm{a} 4$ para ambos os grupos. No $G E$, as pontuações 5 e 6 ocorreram de forma mais expressiva que no GC. Na fase ativa houve predominância, em ambos os grupos, de escores de 6 a 8. No GE, as pontuações 7 e 8 ocorreram de forma mais expressiva do que no GC. Na fase de transição houve predominância dos valores pontuados entre $9 \mathrm{e} 10$, nos dois grupos, com maior frequência para a pontuação igual a $10(70,58 \%$ para o GC e $68,42 \%$ para o GE).

$O$ teste $t$ de Student não mostrou diferenças significativas entre os grupos para as pontuações de intensidade de dor referidas pelas parturientes na fase latente $(p=0,21)$, na fase ativa $(p=0,11) e$ na fase de transição $(p=0,49)$ do trabalho de parto.

O processo fisiológico da dor na parturição está associado à evolução do trabalho de parto. Inicialmente, na fase latente, o padrão da contração uterina é menor. Essa fase destina-se ao apagamento do colo uterino que, em primíparas, acontece antes da dilatação, e o processo doloroso é menos intenso. Na fase ativa, quando o colo uterino obtém $100 \%$ de apagamento, na maioria dos casos, o padrão da contração uterina aumenta com o objetivo de impulsionar o pólo cefálico sobre o colo do útero para promover sua dilatação e, também, a descida do feto através da pelve materna. Isso determina desconfortos maiores como dores na região abdominal, na região lombar e na região púbica, com sensação de estiramento. Na fase de transição, 0 padrão da contração uterina aumenta, ainda mais, para promover a etapa final de descida do feto pela pelve. A parturiente começa a sentir a pressão do pólo cefálico sobre a região perineal e a sensação de que irá ocorrer a expulsão do feto. Nessa fase a dor é mais intensa devido à potência das contrações, necessárias à expulsão do feto.

Durante a primeira e a segunda fase do trabalho de parto a dor é predominantemente mediada por mecanismos de distensão do 
segmento uterino com a contribuição dos mecanismos de dilatação da cérvice e da própria contração uterina. Na fase final do trabalho de parto, a dor já é mais localizada no períneo, ânus e reto, podendo, também, ser sentida nas coxas e pernas ${ }^{(17)}$.

As médias dos valores da intensidade de dor, pontuadas pelas parturientes na escala analógica visual, durante as três fases do trabalho de parto, estão apresentadas na Figura 1.

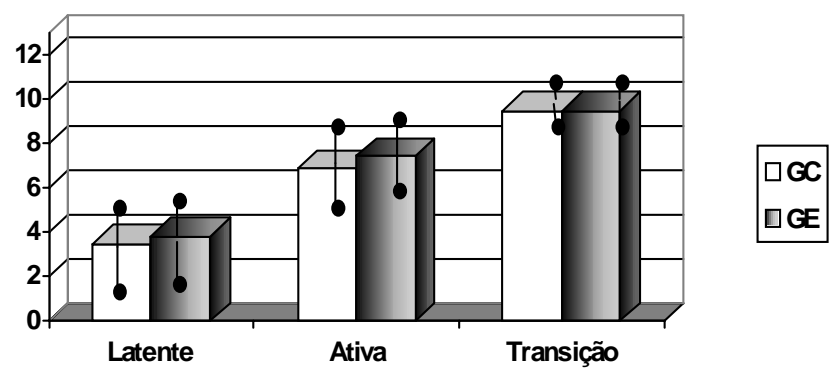

Figura 1 - Valores médios da intensidade da dor $( \pm d p)$ pontuada pelas parturientes dos grupos controle (GC) e experimental (GE) na escala analógica visual, durante as fases do trabalho de parto. Goiânia, GO. 2000/2001

Conforme a Tabela 1 e a Figura 1, a intensidade de dor aumentou gradativamente no decorrer das fases do trabalho de parto, seguindo o padrão fisiológico do seu fator gerador, a contratilidade uterina. Desta forma, foi menor na fase latente e maior na fase de transição, em ambos os grupos.

A média da intensidade de dor referida pelo GC na fase latente foi $3,47( \pm 1,50)$ e pelo $G E$ foi de $3,84( \pm 1,30)$. Na fase ativa, o GC apresentou média de dor referida igual a $6,88( \pm 1,36)$ e o GE $7,42( \pm 1,21)$. Na fase de transição o GC apresentou média de dor referida igual a $9,52( \pm 0,87)$ e o GE $9,52( \pm 0,84)$.

Alguns autores, nos Estados Unidos da América, ao avaliarem a intensidade de dor referida por primíparas, através da escala analógica visual (VAS), obtiveram média igual a $6,0( \pm 2,24)$ no período de 2 a 5 centímetros de dilatação e $8,0( \pm 2,14)$ no período de 6 a 10 centímetros $^{(18)}$. Outros autores, na ltália, ao pesquisarem a intensidade de dor através da VAS, em um grupo de parturientes primíparas e secundíparas, que não utilizaram analgesia, obtiveram intensidade média de dor igual a 5,14 na fase latente, 8,23 na fase ativa e 6,99 na fase de transição $0^{(14)}$.

Ao comparar os achados da literatura com os resultados obtidos no presente estudo, percebe-se que, na fase latente e ativa do trabalho de parto, a média de escores da intensidade de dor foi inferior enquanto na fase de transição a média de escores foi superior.

Isso reforça a idéia de que a dor, durante o trabalho de parto, sofre interferência não somente da contratilidade uterina, mas, também, do contexto sociocultural e psicoafetivo da parturiente ${ }^{(2)}$. Acredita-se que mais estudos necessitam ser realizados a fim de avaliar outros componentes da dor, além da sua intensidade, e garantir 0 planejamento, implementação e avaliação adequada da assistência à parturiente.

Traço de ansiedade e estado de ansiedade

A Tabela 2 mostra um perfil de média ansiedade-traço das parturientes, de ambos os grupos, à admissão no pré-parto, quanto às variáveis grau (G), intensidade (I) e nível de ansiedade (GxI), obtidas através do inventário de ansiedade-traço (IDATE). 0 teste de MannWhitney não mostrou diferença estatisticamente significativa entre as variáveis grau $(p=0,31)$, intensidade $(p=0,83)$ e nível de ansiedade $(p=0,60)$ para o traço de ansiedade dos grupos estudados.

Tabela 2 - Médias do grau (G), intensidade (I) e nível de ansiedade ( $G$ $\mathrm{xI}$ ), obtidas a partir do traço de ansiedade, pontuados pelas parturientes no inventário de ansiedade-traço (IDATE A-traço) ${ }^{\star}$, à admissão no pré-parto. Goiânia, GO. 2000/2001

\begin{tabular}{ccccccc}
\hline Traço de & \multicolumn{3}{c}{$\mathrm{GC}(\mathrm{n}=17)$} & & \multicolumn{3}{c}{$\mathrm{GE}(\mathrm{n}=19)$} \\
Ansiedade & $\mathrm{G}$ & $\mathrm{I}$ & $\mathrm{G} \times \mathrm{I}$ & $\mathrm{G}$ & $\mathrm{I}$ & $\mathrm{G} \times \mathrm{I}$ \\
\hline Média & 43,35 & 106,08 & 238,22 & 41,16 & 106,27 & 227,07 \\
Desvio Padrão & 9,25 & 29,90 & 73,94 & 8,64 & 26,69 & 73,93 \\
\hline
\end{tabular}

* inventário aplicado imediatamente após a admissão

Na Tabela 3 estão apresentadas as médias das variáveis grau, intensidade e nível de ansiedade, pontuados pelas parturientes do GC e do GE nas fases do trabalho de parto e no pós-parto imediato.

Tabela 3 - Médias do grau (G), da intensidade (I) e do nível de ansiedade $(G \times I)$, pontuados pelas parturientes no inventário estado de ansiedade (IDATE A-estado), na admissão, nas três fases do trabalho de parto e no pós-parto imediato. Goiânia, GO. 2000/2001

\begin{tabular}{|c|c|c|c|c|c|c|}
\hline & \multicolumn{3}{|c|}{$\mathrm{GC}$} & \multicolumn{3}{|c|}{ GE } \\
\hline & G & I & $G \times I$ & G & 1 & GxI \\
\hline \multicolumn{7}{|l|}{ Fase latente } \\
\hline Média & $46,94^{\mathrm{abc}}$ & 103,85 & 246,10 & $50,26^{A}$ & 97,88 & $250,31^{a}$ \\
\hline Desvio Padrão & 9,62 & 34,19 & 89,07 & 7,68 & 23,45 & 68,46 \\
\hline \multicolumn{7}{|l|}{ Fase ativa } \\
\hline Média & $54,23^{\mathrm{aD}}$ & 105,91 & $289,50^{A}$ & $52,73^{B}$ & 98,72 & $260,11^{b}$ \\
\hline Desvio Padrão & 7,39 & 25,74 & 81,66 & 2,27 & 21,91 & 79,08 \\
\hline \multicolumn{7}{|l|}{ Fase transição } \\
\hline Média & $54,58^{\mathrm{bE}}$ & 108,99 & $309,31^{B}$ & $55,05^{c}$ & 105,72 & $298,25^{c}$ \\
\hline Desvio Padrão & 8,23 & 34,29 & 121,92 & 7,92 & 28,57 & 113,76 \\
\hline \multicolumn{7}{|l|}{ Pós-parto } \\
\hline Média & $35,64 \mathrm{CDE}$ & 107,31 & $193,02^{A B}$ & $36,84 \mathrm{ABC}$ & 108,51 & $196,07 \mathrm{abc}$ \\
\hline Desvio Padrão & 5,64 & 39,49 & 59,46 & 5,93 & 38,45 & 61,03 \\
\hline
\end{tabular}

Considerando-se a média aritmética de cada uma das variáveis do estado de ansiedade ao longo do processo de parturição, o teste de Mann-Whitney não mostrou diferença estatisticamente significativa para o grau $(p=0,73)$, intensidade $(p=0,55)$, e nível de 
ansiedade $(p=0,60)$ durante o trabalho de parto e o pós-parto imediato entre o GC e GE.

O grau do estado de ansiedade das parturientes nas fases latente, ativa e de transição foi médio para ambos os grupos. No pósparto imediato, o grau de estado de ansiedade apresentou-se baixo.

$\mathrm{O}$ teste de Mann-Whitney mostrou que houve diferença estatisticamente significativa do grau de ansiedade apresentado pelo GC ao comparar a fases do trabalho de parto e o pós-parto imediato, bem como ao se comparar a fase latente e a fase ativa e a fase latente e a de transição. Não houve diferença entre as fases ativa e de transição. Assim pode-se dizer que o grau de ansiedade aumentou na fase ativa, manteve-se constante na fase de transição e diminuiu no pós-parto.

Com relação ao GE, não houve diferenças estatisticamente significativas do grau de ansiedade entre as fases do trabalho de parto. Contudo, houve diferenças estatisticamente significativas entre as fases do trabalho de parto e pós-parto imediato. Assim, pode-se dizer que o grau de ansiedade permaneceu constante no GE ao longo das fases latente, ativa e de transição, diminuindo no pós-parto.

Esses resultados são semelhantes aos de outros pesquisadores que estudaram o grau do estado de ansiedade através do IDATE (State Anxiety Inventory), em um grupo de primíparas e secundíparas, encontrando médias do grau de ansiedade iguais a 45,64 na fase latente, 52,64 na fase ativa, 54,0 na fase de transição e 39,07 no pós-parto ${ }^{(14)}$.

Quanto à intensidade, as parturientes dos dois grupos apresentaram média ansiedade em todas as fases do trabalho de parto, inclusive no pós-parto imediato. Não houve diferença significativa entre as fases do trabalho de parto e entre essas e o pós-parto imediato (Tabela 3).

Ao analisar o nível do estado de ansiedade (produto do grau pela intensidade) para o GC, obteve-se nível baixo de ansiedade na fase latente do trabalho de parto, médio na fase ativa e de transição e baixo no pós-parto imediato. Obteve-se diferença significativa nos níveis de ansiedade entre a fase ativa e o pós-parto imediato e entre a fase de transição e o pós-parto imediato.

De acordo com a pontuação das escalas de ansiedade ${ }^{(13)}$, pode-se dizer que o nível de ansiedade-estado do GC aumentou da fase latente para a ativa, contudo, não houve diferença significativa do nível de ansiedade, comparando-se as duas fases. Observou-se redução do nível de ansiedade no pós-parto, obtendo-se diferença significativa em relação à fase ativa e de transição.

Com relação ao GE, obteve-se baixo nível de ansiedadeestado nas fases latente e ativa, passando para médio na fase de transição e baixo no pós-parto imediato. De acordo com a pontuação das escalas de ansiedade ${ }^{(13)}$, pode-se dizer que o nível de ansiedade aumentou da fase ativa para a de transição, contudo, não houve diferença significativa do nível de ansiedade comparando-se as duas fases. Observou-se a redução do nível de ansiedade no pós-parto e obteve-se diferença significativa em relação a todas as fases do trabalho de parto.

$\mathrm{Na}$ fase de transição é esperado o aumento do nível de ansiedade da parturiente, em decorrência do maior padrão das contrações uterinas e por constituir, no momento, de maior expectativa de resolução do processo de parturição. No pós-parto imediato, essa expectativa termina e cessa a dor proveniente das contrações uterinas. A experiência clínica mostra que esses eventos fisiológicos contribuem para a diminuição do estado de ansiedade.

O teste de Pearson não mostrou correlação entre o grau de ansiedade e a intensidade de dor durante o processo de parturição para o GC, sendo na fase latente $r=-0,11$ e $p=0,67$, na fase ativa $r=$ 0,22 e $p=0,38$ e na fase de transição $r=-0,13$ e $p=0,61 ; 0$ GE apresentou na fase latente $r=-0,11$ e $p=0,64$, na fase ativa $r=0,20$ e $p=0,39$ e na fase de transição $r=-0,12$ e $p=0,62$. Entre a intensidade de ansiedade e a intensidade de dor o GC apresentou na fase latente $r=-0,03$ e $p=0,90$, na fase ativa $r=-0,02$ e $p=0,92$ e na fase de transição $r=0,05$ e $p=0,84 ; 0$ GE apresentou na fase latente $r=0,03$ e $p=0,88$, na fase ativa $r=-0,30$ e $p=0,20$ e na fase de transição $r=-0,27$ e $p=0,25$. Entre o nível de ansiedade e a intensidade de dor o GC apresentou na fase latente $r=-0,10$ e $p=0,69$, na fase ativa $r=0,12$ e $p=0,05$ e na fase de transição $r=-0,03$ e $p=0,90 ; 0$ GE apresentou na fase latente $r=-0,10$ e $p=0,66$, na fase ativa $r=$ $0,13, p=0,58$ e na fase de transição $r=-0,25$ e $p=0,28$.

Esses resultados corroboram com outro achado ${ }^{(19)}$, no qual a escala analógica visual foi utilizada para avaliar a intensidade da dor e o IDATE o estado ansiedade. Os autores não observaram correlação significativa entre os escores de dor referidos pelas parturientes e aqueles do estado de ansiedade. Também não observaram correlação significativa entre a preparação psicológica para o parto e a ansiedade, na admissão ou entre a avaliação de dor e a duração do trabalho de parto.

\section{CONCLUSÕES}

Em relação à intensidade de dor referida pelas parturientes, os achados apontam escores médios como pontuação crescente durante o trabalho de parto, sendo menor a intensidade média de dor na fase latente (GC - 3,47 e GE - 3,84) e maior na fase de transição (GC e GE -9,52), sem diferenças estatisticamente significativas entre os grupos.

O nível de ansiedade, na fase latente, foi baixo para ambos os grupos, na fase ativa foi médio para o GC e baixo para o GE, na fase de transição foi médio e no pós-parto imediato baixo, para ambos os grupos. Não houve diferença estatisticamente significativa do grau, 
da intensidade e do nível de ansiedade entre os grupos.

Não houve correlação entre as variáveis do estado de ansiedade e a intensidade de dor nas fases do trabalho de parto para ambos os grupos.
Concluiu-se que as técnicas utilizadas não reduziram a intensidade de dor, mas promoveram ao GE a manutenção de baixo nível de ansiedade-estado por maior tempo na parturição (fase latente e ativa).

\section{REFERÊNCIAS BIBLIOGRÁFICAS}

1. Pimenta CAM, Portnoi AG. Dor e Cultura. In: Carvalho MMMJ, organizadora. Dor: um estudo multidisciplinar. São Paulo (SP): Summus Editora; 1999. p.159-73.

2. Lowe K N. The nature of labor pain. Am J Obstet Gynecol 2002; 186:S16-24.

3. Guyton AC, Hall JE. Tratado de fisiologia médica. 10ª ed. Rio de Janeiro (RJ): Guanabara Koogan; 2002.

4. Guimarães SS. Introdução ao estudo da dor. In: Carvalho MMMJ, organizadora. Dor: um estudo multidisciplinar. São Paulo (SP): Summus Editora; 1999. p.13-30.

5. Burroughs A. Uma introdução à enfermagem materna. 6aㅡ ed. Porto Alegre (RS): Artes Médicas; 1995.

6. Maldonado MT. Psicologia da gravidez, parto e puerpério. $12^{\mathfrak{a}}$ ed. Petrópolis (RJ): Editora Vozes; 1991.

7. Teixeira MJ, Pimenta CAM, Crossi SAA, Cruz DLM. Avaliação da dor: fundamentos teóricos e análise crítica. Rev Médica 1999; 78(2 Pt.1):85-114.

8. Hodnett ED. Pain and women's satisfaction with the experience of childbirth: A systematic review. Am J Obstet Gynecol 2002; 186:S16072.

9. Pimenta CAM, Teixeira MJ. Avaliação da dor. Rev Médica 1997 jan/ fev; 76(1):27-35.

10. Melzack R, Katz F. Pain measurement in persons in pain. In: Wall PD, Melzack R, organizadores. Textbook of pain. $3^{\text {rd }}$ ed. New York (USA):Churchill Livingstone; 1994. p.337-51.

11. Browndige $P$. The nature and consequences of childbirth pain. Eur J Obstet Gynecol Reprod Biol 1995; (59 Suppl):S9-S15.

12. Spielberger CD, Gorsuch RL, Lushene RE. Inventário de ansiedade traço-estado: IDATE (STATE-TRAIT ANXIETY INVENTORY - STAI). Rio de Janeiro (RJ): Centro de Estudos de Psicologia Aplicada (CEPA); 1979.

13. Amorin MHC. A enfermagem e a psiconeuroimunologia no câncer de mama. [Tese]. Rio de Janeiro (RJ): Escola de Enfermagem Anna Nery/ UFRJ; 1999.

14. Caton D; Corry MP; Frigoletto FD; Hopkins DP; Lieberman E; Mayberry $L$, et al. The nature and management of labor pain: executive summary. Am J Obstet Gynecol 2002; 186:S1-15.

15. Pancheri P, Zichella L, Fraioli F, Carilli L, Perrone G, Biondi M, et al. ACTH, Beta-endorphin and met-enkephalin: peripheral modifications during the stress of human labor. Psychoneuroendocrinology 1985; 10(3):289-301.

16. Lucas KH. Facilitação psicológica do parto. $3^{\underline{a}}$ ed. São Paulo (SP): Manole; 1983.

17. Rowlands S, Permezel M. Physiology of pain in labour. Baillieres Clin Obstet Gynecol 1998 Sep; 12(3):347-362.

18. Brown ST, Campbell D, Kurtz A. Characteristics of labor pain at two stages of cervical dilation. Pain 1989; 38:289-95.

19. Bonnel AM, Boureau F. Labor pain assessment: validity of a behavioral index. Pain 1985; 22:81-90. 